\title{
La violencia social delincuencial asociada a la salud mental en los salvadoreños
}

\author{
José Ricardo Gutiérrez Quintanilla \\ ricardo.gutierrez@utec.edu.sv \\ Dirección de Investigaciones. \\ Universidad Tecnológica de El Salvador
}

\section{Resumen}

El presente estudio tiene como objetivo general determinar si existe una relación de la violencia social delincuencial con la salud mental en la población salvadoreña. Para ello se operacionalizó y construyó un instrumento que midiese el estrés y la ansiedad delincuencial, como indicadores de afectación de la salud mental en la población víctima de la violencia delincuencial. En el estudio se utilizó un muestreo probabilístico por conglomerados a escala nacional en una muestra de 1.143 personas, de estas 605 (52,8\%) son mujeres y $538(47,1 \%)$ son hombres. El grupo de edad mayoritario está entre 26 a 35 años (26,8\%). Es un estudio que puede ser tipificado como multimodal (Hernández, Fernández y Baptista, 2006). También podría nominarse ex post facto (Montero y León, 2007), con un diseño retrospectivo y transeccional. Las técnicas utilizadas para la recolección de información fueron la encuesta y la entrevista en profundidad. Se construyeron y validaron para medir las variables del estudio la Escala de estrés y ansiedad delincuencial (ESAD) y la Escala de ambiente familiar (ESAF); se adaptó el Cuestionario

\section{Abstract}

This study aimed to determine whether there is a relationship between social criminal violence and mental health in the Salvadoran population. For this purpose, an instrument was built and operationalized so it would measure the crime stress and anxiety as indicators of impaired mental health in the victims of criminal violence. The study used a random, national sample of 1.143 people. Of these $605(52,8 \%)$ were women and 538 $(47,1 \%)$ were men. The majority age group is between 26 to 35 years $(26,8 \%)$. The study type is multimodal (Hernandez Fernandez and Baptista, 2006). It can also be named ex post facto (Montero and León, 2007), with a retrospective transversal design. The techniques used for collecting information were surveys and indepth interviews. To measure the variables of the study of stress and anxiety, the scale crime (ESAD) and the family environment scale (ESAF) were constructed, and the General Health Questionnaire, GHQ-12 was adapted (Golbert, 1970). All these instruments have reliability and validity in El Salvador. The study reveals that there is increased incidence of crime stress and anxiety, and 
de salud general, GHQ-12 (Golbert, 1970). Todos estos instrumentos gozan de fiabilidad y validez en El Salvador. El estudio revela que existe mayor incidencia de estrés $\mathrm{y}$ ansiedad delincuencial, y pobre salud mental, en las mujeres y en los residentes urbanos. El modelo de regresión logística demuestra que la ansiedad delincuencial en la población salvadoreña es explicada en un porcentaje importante por las variables: estresor delincuencial, violencia sociocultural, deterioro de la salud mental y el sexo de la muestra.

Palabras clave: Salud mental, violencia social delitos, estrés-ansiedad delincuencial, conducta colectiva, funcionamiento familiar. poor mental health in women and urban residents. The logistic regression model shows that crime anxiety in the Salvadoran population is explained in a significant percentage by the variables: criminal stressor, cultural violence, deterioration of mental health and sex of the sample.

Keywords: Mental health, social violence crimes, criminal Stress-anxiety, collective behavior, family functioning.

\section{Introducción}

La violencia social delincuencial es definida como el contexto comunitario donde vive una sociedad, caracterizado por la frecuente presencia de diferentes tipos de delitos como: homicidios, lesionados, extorsiones, robos, asaltos y secuestros, cometidos por grupos delincuenciales, por ejemplo: las pandillas, los narcotraficantes, grupos de sicarios, etc. En general, estos tipos de delitos son cometidos en las colonias, los barrios, en los autobuses, en las calles, en las plazas y parques; eventos que con el paso del tiempo van afectando la estabilidad emocional y mental de las personas que los experimentan; sus efectos pueden manifestarse en temor, fobias, insomnio, estrés, ansiedad, depresión, y en algunos casos llegar a estrés postraumático y otras alteraciones mentales.

En el año 2009 ocurrieron 4.382 homicidios, mientras que en el año 2010 hubo 3.985 homicidios (Policía Nacional Civil. PNC, 2010), siendo la población general salvadoreña víctima constante del acecho de estos grupos delincuenciales. Es por ello que el investigador se trazó como objetivo general determinar si el contexto social delincuencial en que viven los salvadoreños está relacionado con la salud mental y otras variables, como el ambiente familiar, el consumo de drogas y alcohol, entre otras. Este contexto de violencia social delincuencial en que vive permanentemente la población salvadoreña motivó al equipo a formularse algunas hipótesis generales sobre el problema. Entre ellas se tienen: "La violencia social delincuencial es un factor que está asociado a la sensación de inseguridad y a la pérdida de confianza en el sistema", "El contexto de violencia social delincuencial está relacionado con los niveles de estrés social de la población", "el estrés social delincuencial está asociado con la ansiedad, la salud mental, la estabilidad emocional familiar y comunitaria de la población salvadoreña".

En estudios realizados en la población salvadoreña se ha encontrado alta incidencia (arriba del 50\%) de alteraciones mentales, como ansiedad, disfunciones sociales y trastornos psicosomáticos (Gutiérrez, 2010). Estos problemas mentales fueron asociados a variables sociodemográficas como la edad, el sexo, lugar de residencia (urbano o rural), nivel educativo, entre otras. En este mismo sentido, en los últimos cinco años El Salvador ha vivido en un contexto de violencia social delincuencial que, de alguna forma, podría estar relacionado con los problemas y la falta de salud mental que presenta la población. El estrés social que experimenta la población es el resultado de factores psicosociales como el desempleo, la falta de oportunidades, la violencia delincuencial, la insatisfacción de sus necesidades básicas, que son variables que están vinculadas a la salud mental. En 
consecuencia, los resultados de la violencia delincuencial podrían estar incrementando en la población salvadoreña las alteraciones como ansiedad generalizada, temor, fobias, signos de depresión e insomnio; síntomas que pueden ser el resultado de la exposición contante a un estrés social delincuencial. En este estudio se pretende establecer si existe el estrés social delincuencial, y si está asociado con indicadores de problemas de salud mental en la población salvadoreña.

\section{La violencia y la salud mental}

Según la Organización Mundial de la Salud (OMS. 2003), la prevención de la violencia ha sido un tema prioritario en los últimos años, de tal manera que debe ser abordada desde el enfoque de la salud pública, lo que implica un tratamiento desde diversos ámbitos para reducir sus niveles de incidencia. Cada año, en el mundo, más de 1.6 millones de personas pierden la vida de forma violenta y muchas otras sufren lesiones no mortales como resultado de la violencia, sea esta autoinfligida, interpersonal o colectiva. Las modernas concepciones de salud fueron reunidas en la década de los setenta a través de la OMS y formuladas a raíz de la Conferencia de Alma-Atá. A partir de este momento se define la salud como una yuxtaposición de grados de bienestar en los campos psíquico, físico y social, y no solo como ausencia de enfermedad. Los diferentes grados de salud permanecen vinculados a variables biológicas, psicológicas y del entorno. Se podría pensar que la atención a la salud y a la enfermedad mental sigue centrada en la patología del cerebro y del sistema nervioso. Sin embargo, existen grades aportes de la psicología en sus diferentes especialidades y de la sociología. Reyes (2007), en su estudio, considera que tener una enfermedad mental es un hecho que influye en las reacciones violentas. Resulta interesante que los pacientes, familiares y los vecinos allegados expresan que sí influye la condición de estar enfermos en la aparición de la violencia; esto surge por la dificultad que presenta la persona en la represión de sus impulsos, pero señalan que paradójicamente son más agredidos los enfermos que lo que ellos son capaces de agredir, estos se reconocen como víctimas porque, a pesar de estar enfermos, tienen que seguir interactuando en la sociedad con sus limitaciones.
En el estudio MacArthur (1998) se observaron dos predictores de la conducta violenta: uno, la psicopatía, y dos, el haber sido víctima de malos tratos durante la infancia. Este mismo estudio encontró que la tasa de violencia fue significativamente superior en los esquizofrénicos y en aquellos que eran consumidores de sustancias psicoactivas y/o alcohol. La enfermedad mental ha sido un factor asociado tradicionalmente a la delincuencia (Núñez y López, 2009) pues existen determinados comportamientos criminales que pueden relacionarse $o$ atribuirse a anomalías mentales. Es preciso tener en cuenta que no todo criminal o delincuente es un enfermo mental, ni que todo enfermo mental comete actos delictivos, aunque exista un diagnóstico clínico debe existir una relación de causalidad con el acto (Sánchez Gutiérrez, 2000). Garrido y López (2006) afirman que el hecho de que alguien desafíe los principios esenciales que regulan nuestra vida social, forjados a lo largo de siglos, no es prueba o razón suficiente para pensar que sean locos, enfermos o degenerados. A pesar de los numerosos estudios llevados a cabo para relacionar la delincuencia y los trastornos psicopatológicos, no pueden extraerse conclusiones definitivas, debido a que muchas de estas investigaciones se han realizado con delincuentes privados de libertad, circunstancia que puede favorecer la aparición de ciertos trastornos mentales, como alteraciones emocionales, trastorno límite de la personalidad y trastornos disociativos (Luberto, Zavatti y Gualandri, 1997).

\section{Estrés general}

El estrés supone un hecho habitual de la vida del ser humano, ya que cualquier individuo, con mayor o menor frecuencia, lo ha experimentado en algún momento de su existencia. El más mínimo cambio al que se expone una persona es susceptible de provocárselo. Tener estrés es estar sometido a una gran presión, sentirse frustrado, aburrido, encontrarse en situaciones en las que no es fácil su control, tener problemas conyugales, etc. El origen del término estrés se encuentra en el vocablo distres, que significa en inglés antiguo "pena o aflicción"; con el uso frecuente se ha perdido la primera sílaba. El vocablo ya era usado en física por Selye (1936), aludiendo a la fuerza que actúa sobre un objeto produciendo su destrucción al superar una determinada magnitud. En 1936, Hans Selye introdujo el término estrés como un síndrome específico 
constituido por cambios inespecíficos del organismo inducidos por las demandas del entorno. Para este autor, el estrés es una respuesta inespecífica del organismo ante una diversidad de exigencias. Se trata de un proceso adaptativo y de emergencia, siendo imprescindible para la supervivencia de la persona, este no se considera una emoción en sí mismo, sino que es el agente generador de las emociones. En todo caso, el estrés es una relación entre la persona y el ambiente, en donde el sujeto percibe en qué medida las demandas ambientales constituyen un peligro para su bienestar, si exceden o igualan sus recursos para enfrentarse a ellas (Lazarus y Folkman, 1984).

Desde esta perspectiva, los autores manejan diversos datos, unos se relacionan más con la rama de la Fisiología, y otros, con la Psicología, siendo esta última la que engloba un mayor número de manifestaciones en el sujeto, dado que los individuos responden a cualquier demanda del entorno, incluyendo las de naturaleza psicosocial. Si el sentimiento de carácter negativo aparece de forma constante en el sujeto y no es tratado adecuadamente, puede conducir a un bajo rendimiento en la vida cotidiana, debilitando la salud (Olga y Terry, 1997). Casi todas las personas han oído hablar del estrés, pues es este un término que utilizan por igual profesionales de la salud y profanos en la materia; para unos es sinónimo de sobresalto, para otros hace referencia a malestar, y, para la mayoría, un generador de tensión para el sujeto. No obstante, en todos estos casos, el estrés es entendido como algo negativo, perjudicial o nocivo para el ser humano, ya que produce dolores de cabeza, indigestión, resfriados frecuentes, dolor de cuello y espalda e infelicidad en las relaciones personales más cercanas (Olga y Terry, 1997); además, el estrés puede incapacitar al individuo en el ámbito laboral, provocar crisis nerviosas recurrentes, depresión, ansiedad o incluso dar lugar a la muerte por un ataque al corazón. La vivencia del estrés como positivo o negativo dependerá de la valoración que realiza el individuo de las demandas de la situación y de sus propias capacidades para hacerles frente. Según esto, el ser humano se enfrenta continuamente a las modificaciones que va sufriendo el ambiente, percibiéndolas y reinterpretándolas con el objeto de poner en marcha conductas en función de dichas interpretaciones. Hay ocasiones en las que la demanda del ambiente es excesiva para el individuo, por lo que su repertorio conductual para hacer frente a la situación generadora de estrés es insuficiente, al igual que cuando se enfrenta a una situación nueva para él (Olga y Terry, 1997).

\section{La ansiedad}

Los estudios sobre la ansiedad se han desarrollado a lo largo de la historia con dos problemas fundamentales: la ambigüedad conceptual del constructo de ansiedad y las dificultades metodológicas para abordar los estudios. Estos problemas dieron lugar a que las distintas corrientes psicológicas (psicodinámica, humanista, existencial, conductista, psicométrica y la cognitiva-conductual) se ocuparan del abordaje de la ansiedad y de las similitudes y diferencias con otros conceptos, dada la gran confusión terminológica con la angustia, el estrés, el temor, el miedo, la tensión arousal, entre otros. Esta confusión conceptual ha sido objeto de diversos estudios (Ansorena, Cobo y Romero, 1983; Bermúdez y Luna, 1980; Borkovek, Weerts y Berstein, 1977; Casado, 1994; Cattell, 1973; Lazarus, 1966; MiguelTobal, 1985). En la práctica actual dichos términos se siguen utilizando indistintamente.

En la actualidad es difícil mantener dichas diferencias, ya que dentro del concepto de ansiedad agrupamos tanto los síntomas psíquicos, o cognitivos, como los conductuales y físicos. Además, en el DSM-IV (Manual de clasificación de trastornos psicopatológicos) el trastorno de ansiedad se denomina trastorno de angustia, resaltando, entre otras características, la presencia de crisis de angustia recidivantes e inesperadas (American Psychiatric Association, 1994). Durante la década de los años cincuenta, y posteriormente en los sesenta, la investigación psicológica estaba centrada en el trastorno mental de la esquizofrenia; mientras que en los años setenta el interés se dirigió a la evaluación de los estados de ánimo, especialmente la depresión. Sin embargo, en 1985 dos psicólogos norteamericanos, Husain y Maser, afirmaron que la década de los años ochenta pasaría a la historia como la década de la ansiedad; y es a partir de entonces cuando esta pasa a ocupar un lugar preferente que perdura hasta nuestros días. En esta época, las neurosis de ansiedad se caracterizan por una preocupación ansiosa exagerada que llega hasta el pánico y va acompañada a menudo por síntomas somáticos. La neurosis de ansiedad (al contrario que la neurosis fóbica) puede producirse en cualquier circunstancia y no está limitada a situaciones 
u objetos específicos (DSM II, 1986). Actualmente, los trastornos de ansiedad ocupan el primer lugar a escala mundial entre los trastornos del comportamiento más prevalentes.

Las relaciones entre ansiedad y salud mental parecen estar bastante bien establecidas, sobre todo en la adolescencia (por ejemplo, Axelsson y Ejlertsson, 2002; Bagley y Mallick, 2001; Chamberlain y Haaga, 2001). Los autores coinciden en señalar que la ansiedad afecta de modo directo a la salud mental de las personas que la sufren. Se han encontrado relaciones entre la ansiedad y los estilos de vida que promocionan la salud (Johnson, 2002). Además, la ansiedad se ha revelado asociada al riesgo de desarrollar ciertas patologías, como por ejemplo, la hipertensión en las mujeres (Adler y Matthews, 1994), o como factor de riesgo de la sobremortalidad cardiovascular (Hansen, 2003). En estudio realizado recientemente a escala nacional (Gutiérrez, 2010) se encontró prevalencia de ansiedad de $53,6 \%$ y alteraciones psicosomáticas de $60,0 \%$. Además de la existencia de casos de psicopatología en $35,1 \%$ de la población general. Son estos hallazgos y demás factores psicosociales los que han motivado al estudio de las variables predictoras de la falta de salud mental; entre estas se pueden mencionar: el estrés social, la ansiedad, la violencia delincuencial, el ambiente familiar, el contexto sociocultural, el consumo de drogas y alcohol y la salud mental de la población. Ante los potenciales efectos psicológicos y emocionales de la violencia delincuencial en la salud mental de los salvadoreños surgen los interrogantes: ¿Existirá una relación del contexto de violencia social delincuencial con la salud mental de la población salvadoreña? ¿Qué tipos de violencia delincuencial afectan más la salud mental de los salvadoreños? ¿Habrá algún tipo de relación de las variables socioculturales con la salud mental de los salvadoreños? ¿Existirá alguna asociación de las variables sociodemográficas con la estabilidad mental y emocional de los salvadoreños? Con la finalidad de sistematizar el desarrollo del presente estudio y responder algunas de las preguntas antes señaladas, se plantean los siguientes objetivos de investigación:

1. Determinar si existen diferencias significativas al comparar el estrés-ansiedad delincuencial, la salud mental en función del sexo y el lugar de residencia de la población.
2. Determinar si existe una asociación del estrés y la ansiedad delincuencial con la salud mental de los salvadoreños.

\section{Método}

\section{Participantes}

En este te estudio se seleccionó una muestra probabilística representativa de las ciudades más importantes del país. Para ello, se adopto una estimación de error del 3\% y un nivel de confianza del 95\%, obteniéndose una muestra de 1.143 personas a escala nacional. La muestra fue distribuida de forma proporcional al número de habitantes en cada departamento. El presente estudio es de tipo multimodal (Hernández, Fernández y Baptista, 2006); también podría nominarse ex post facto (Montero y León, 2007), con un diseño retrospectivo y transeccional. Entre las técnicas para la recolección de información fueron utilizadas: la encuesta y la entrevista con profundidad. En el estudio fueron encuestadas $538(47,1 \%)$ hombres y $605(52,9 \%)$ mujeres. Siendo esta una muestra que está en sintonía con el universo de la población salvadoreña. En cuanto a la edad, el mayor porcentaje está entre 25 a 36 (26,8\%) años. En relación con la variable lugar de residencia 807 (70,6\%) fueron urbanas y $336(29,4 \%)$ rurales, las variables como el nivel educativo, estado civil, lugar de residencia.

\section{Instrumentos}

\section{Escala de estrés y ansiedad delincuencial (ESAD).}

Tras la construcción de la escala, se procedió a administra la prueba a una muestra de 300 personas, siendo este el primer estudio de cara a los análisis psicométricos de la prueba. Originalmente la escala quedó integrada por diecisiete ítems. Tras las pruebas psicométricas mediante el Análisis Factorial Exploratorio (AFE) este arrojó dos factores integrados por: el estresor delincuencial y ansiedad delincuencial. El primero era evaluado con los primeros cinco ítems $(1,2,3,4,5)$, y el segundo con los ocho ítems siguientes $(6,7,8,9,10,11,12$, 13). También se aplicó el análisis de consistencia interna; sus alfa de Cronbach fueron superiores a 0,75 , indicando adecuados criterios de fiabilidad y validez de constructo en ambas dimensiones. Quedando un instrumento con 
buenas propiedades psicométricas para evaluar el estrés y ansiedad delincuencial en una población salvadoreña. Posterior al estudio dos realizado a escala nacional, se procedió a repetir los mismos análisis psicométricos encontrándose los resultados siguientes; en el Análisis Factorial Exploratorio mediante el análisis Kaiser-MayeOlkin $(\mathrm{KMO}=0,86)$ con la prueba de esferidad de Bartlett $\left(X^{2} 78=5235 ; p=0,000\right)$ indicaron la adecuación de los datos para este tipo de análisis. Siguiendo el procedimiento prefijado en dos factores con rotación Varimax, resultando una solución de dos factores que explican el 49,99\% de la varianza total explicada, presentando todos los ítems valores de saturación superiores a 0,40; siendo el primer factor: Estresor delincuencial con seis ítems (1, 2, 3, 4, 5, 6), su alfa de Cronbach es de 0,77 ; y el segundo factor: ansiedad delincuencial, con siete ítems $(7,8,9,10,11,12$, 13), el alfa de Cronbach es de 0,82 , indicando que la prueba posee adecuadas propiedades psicométricas para evaluar este constructo o variable latente.

\section{Cuestionario de salud general, GHQ-12. (Golbert, 1970)}

Este es un instrumento de screning (filtrado) o de tamizaje de salud mental en la población que evalúe salud mental y bienestar psicológico. La prueba posee adecuadas propiedades psicométricas de fiabilidad y validez. En el estudio cubano se obtuvieron los indicadores de consistencia interna alfa de Cronbach de 0,89 para las dos escalas; en el estudio en muestra española se obtuvieron índices de consistencia interna de 0,82 y 0,85. En el estudio cubano, la validez se logró mediante la correlación con la escala de depresión de Beck, y esta fue de 0,92; y con el Idare de 0,85 . Similares resultados se encontraron en países como Japón, EE.UU. e Inglaterra. Lo anterior indica que la prueba posee adecuadas propiedades psicométricas para evaluar bienestar psicológico (salud mental) y funcionamiento social. En el presente estudio, las pruebas de consistencia interna alfa de Cronbach obtenidas fueron superiores a 0,77 en ambas dimensiones, indicando que la prueba posee adecuadas propiedades psicométricas en El Salvador.

\section{Resultados}

El análisis descriptivo se desarrollará, en un primer momento, del estrés-ansiedad delincuencial resultado de la violencia delincuencial en relación con el sexo y el lugar de residencia (urbano, rural) de la muestra; en un segundo momento, de los indicadores de salud mental/bienestar psicológico, comparado por sexo y lugar de residencia. Asimismo, en función de sí ha sido víctima de violencia delincuencial o no. Posteriormente se presentarán las incidencias de víctimas de violencia, lugar de cometimiento del delito, el contexto sociocultural de la violencia; y el consumo de drogas y alcohol, las razones y motivaciones del consumo, la frecuencia y tipos de drogas, entre otras variables relacionadas.

A continuación se presentan las frecuencias y porcentajes de la muestra que frecuentemente presentan estrés y ansiedad delincuencial comparándose por sexo. En este primer momento, se enfatiza en aquellos indicadores que revelaron diferencias estadísticamente significativas entre hombres y mujeres. En relación con la pregunta si en el último año un pariente suyo fue víctima de la violencia delincuencial: lesión u homicidio: 409 personas $(36,0 \%)$ expresaron que frecuentemente; de estos, 174 (42,5\%) son hombres, mientras 235 (57,5\%) son mujeres, indicando diferencias significativas; mientras $26,6 \%$ de la muestra acepto haber sido víctima de la delincuencia en el último año. En cuanto a si consideran que las noticias que se transmiten por televisión, prensa escrita y radio, sobre la violencia delincuencial (lesionados, muertos, extorsiones) afectan su estado emocional y mental, $868(76,5 \%)$ manifestaron que frecuentemente; de este total $387(44,6 \%)$ son masculinos y $481(55,4 \%)$ son femeninos.

Se preguntó si siente temor de transitar por algunas calles o zonas de la ciudad (colonias, barrios o pueblo) $922(81,3 \%)$ expresaron que frecuentemente, de estos 415 (45,0\%) son masculinos y $507(55,0 \%)$ son del sexo femenino. En relación con sí el contexto de violencia social delincuencial en que viven los salvadoreños le produce ansiedad y estrés (pánico, miedo, agitación, boca seca, inquietud, taquicardia) 866 $(76,4 \%)$ manifestaron que frecuentemente; de este total, $392(45,3 \%)$ son masculinos y $474(54,7 \%)$ son femeninos, encontrándose diferencias significativas entre ambos grupos, presentando mayores dificultades de salud mental las mujeres que los hombres (ver tabla 1). Referente a sí el ambiente de violencia social delincuencial que viven los salvadoreños le produce nerviosismo e inseguridad, 961 
$(84,8 \%)$ dicen que frecuentemente, del total, $436(45,4 \%)$ son masculinos y $525(54,6)$ son femeninos. Al preguntarle a la población si considera usted que la situación de violencia delincuencial que vive el país le afecta el sueño, 751 (65,9\%) refieren que frecuentemente; de estos, 326 (43,4\%) son del sexo masculino y 425 (56,6\%) son femeninos. Si hay diferencias significativas entre ambos grupos, estando más afectadas las mujeres.
En cuanto a sí el contexto de violencia social delincuencial que se vive en El Salvador está afectando su estabilidad emocional y mental, 807 (70,7\%) expresan que frecuentemente; de estos, 347 (43,0\%) son hombres y 460 (57,0\%) son mujeres. En los indicadores restantes, si bien no hay diferencias estadísticamente significativas entre hombres y mujeres, la prevalencia de estrés y ansiedad delincuencial siempre es mayor en la mujer.

Tabla 1.

Frecuencias y porcentajes de la muestra que frecuentemente presentan estrés y ansiedad delincuencial, comparados por sexo

No.

Ítems

¿En el último año, algún pariente suyo fue víctima de la delincuencia (Ej.: robo, hurto, extorsión)?

2

¿En el último año, algún amigo o conocido suyo fue víctima de la delincuencia (Ej.: robo, hurto, extorsión)?

3

¿En el último año, algún pariente suyo fue víctima de la violencia delincuencial (Ej.: lesión u homicidio)?

4 ¿Usted ha pensado en cambiar su lugar de residencia como producto de la violencia delincuencial?

¿Algún familiar, amigo o conocido ha cambiado su lugar de residencia como resultado de la violencia?

6

¿En su casa, se han implantado algunas medidas de seguridad (Ej.: cerrar temprano la puerta, no estar en la calle)?

¿Considera usted que las noticias que transmiten por televisión, 7 prensa escrita y radio sobre la violencias delincuencial (Ej.: lesionados, muertos, extorsiones) afectan su estado emocional y mental?

Total Hombres Mujeres

(\%) (\%) (\%)

498

$(43,8)$

656

$(57,5)$

409

$(36,0)$

427

$(37,9)$

574

$(50,9)$

859

$(76,4)$

868

$(76,5)$
265

233

$(46,8)$

$(53,2)$

324

$(49,4)$

332

$(50,6)$

$174 \quad 235$

$(42,5)$

$(57,5)$

5,316 *

191

$(44,7)$

236

$(55,3)$

1,812

274

$(47,9)$

299

0,233

399

460

$(46,4)$

$(53,6)$

0,639

387

$(44,6)$

481

$(55,4)$
$3,756 \dagger$

0,017

\section{$+$

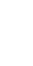


¿En el último año, ha modificado su vida cotidiana debido a la

8 violencia delincuencial (Ej.: Llegar temprano a casa, no salir de noche)?

$\begin{array}{cccc}855 & 397 & 458 & \\ (75,6) & (46,4) & (53,6) & 0,376\end{array}$

9 ¿Siente temor de transitar por algunas calles o zonas de la 922 ciudad (colonias, barrios o pueblo)?

415

$(45,0)$

$(55,0)$

$7,848^{* *}$

¿El contexto de violencia delincuencial que se vive en El Salvador

10 le produce ansiedad y estrés (pánico, miedo, agitación, boca seca, inquietud, taquicardia)?

¿El ambiente de violencia delincuencial que se vive en El Salvador le produce nerviosismo e inseguridad?

12

¿Considera usted que la situación de violencia delincuencial que vive el país le afecta el sueño?

13

¿El contexto de violencia delincuencial que se vive en El Salvador afecta su estabilidad emocional y mental?

$\begin{array}{cccc}866 & 392 & 474 & 5,135 * \\ (76,4) & (45,3) & (54,7) & \end{array}$

961

$(84,8)$

436

$(45,4)$

525

$(54,6)$

$7,119 * *$

$\begin{array}{lll}751 & 326 & 425\end{array}$

$(65,9)$

$(43,4)$

$(56,6)$

$11,506 * *$

807

$(70,7)$
460

$(57,0)$
$17,511 * * *$
${ }^{*} \mathrm{p}<0,05 ; * * \mathrm{p}<0,01 ; * * * \mathrm{p}<0,001$

Con respecto a las frecuencias y porcentajes de la muestra que manifestaron que frecuentemente presentan estrés y ansiedad delincuencial en función del lugar de residencia (urbano, rural), en el presente análisis se considerarán aquellos indicadores que su prevalencia total encontrada fue superior al $75 \%$. Uno de los primeros indicadores es: en su casa se han implantado algunas medidas de seguridad, como cerrar temprano la puerta, no estar en la calle, 859 $(76,4 \%)$ expresaron que frecuentemente lo hacen; de estos, $613(71,4 \%)$ son urbanos y $246(28,6 \%)$ son rurales. En relación con que sí consideran que las noticias que se transmiten por televisión, prensa escrita y radio, sobre la violencia delincuencial (lesionados, muertos, extorsiones) afectan su estado emocional y mental, 868 (76,5\%) del total manifestaron que frecuentemente; de este total, 614 $(70,7 \%)$ son urbanos y $254(29,3 \%)$ son rurales. También se preguntó si en el último año ha modificado su vida cotidiana debido al contexto de violencia delincuencial, Ej.: llegar temprano a casa, no salir de noche; $855(75,6 \%)$ refieren que frecuentemente lo hacen; de estos, $599(70,1 \%)$ son urbanos y $256(29,9 \%)$ son rurales (ver tabla 6). Por otra parte, 866 $(76,4 \%)$ de la muestra del estudio expresaron que el contexto de violencia social delincuencial que se vive en El Salvador les produce ansiedad, estrés, pánico, miedo, agitación, boca seca, inquietud y taquicardia; de este total, $614(70,9 \%)$ son urbanos y $252(29,1)$ son rurales. Asimismo, $961(84,8 \%)$ consideran que el ambiente de violencia delincuencial que se vive en El Salvador les produce nerviosismo e inseguridad; de estos, $674(70,1 \%)$ son urbanos y $287(29,9 \%)$ son rurales.

Todos los indicadores de estrés y ansiedad delincuencial, tanto los antes señalados como el resto no mencionados, presentan una rotunda mayoría de prevalencia en la personas que residen en las ciudades en contraste con las personas que residen en las zonas rurales, indicando claramente que los efectos directos de la violencia social delincuencial son los problemas de salud mental y bienestar psicológico en la población salvadoreña, con mayor significancia en los residentes de la ciudad que en los residentes rurales. 
Tabla 2.

Frecuencias y porcentajes de la muestra que frecuentemente presenta estrés y ansiedad delincuencial, comparando los que residen en zonas urbanas y rurales

No.

Ítems

Total

Urbano

Rural

(\%)

(\%)

(\%)

¿En el último año, algún pariente suyo fue víctima de la delincuencia

(Ej. robo, hurto, extorsión)?

498

$(43,8)$

360

$(72,3)$

138

$(27,7)$

0,983

¿En el último año, algún amigo o conocido suyo fue víctima de la delincuencia (Ej. robo, hurto, extorsión)?

656

$(57,5)$

475

$(72,4)$

181

$(27,6)$

2,173

$\begin{array}{cccc}409 & 293 & 116 & \\ (36,0) & (71,6) & (28,4) & 0,186\end{array}$

delincuencial (Ej. lesión o homicidio)?

$(36,0)$

$(71,6)$

427

$(37,9)$

318

109

producto de la violencia delincuencial?

$(74,5)$

$(25,5)$

$4,469 *$

¿Algún familiar, amigo o conocido ha cambiado su lugar de residencia como resultado de la violencia?

574

$(50,9)$

859

$(76,4)$

(Ej.: cerrar temprano la puerta, no estar en la calle)?

¿Considera usted que las noticias que transmiten por televisión,

7 prensa escrita y radio sobre la violencias delincuencial (Ej.: lesionados, muertos, extorsiones) afectan su estado emocional y mental?

¿En el último año, ha modificado su vida cotidiana debido a la

8 violencia delincuencial (Ej. Llegar temprano a casa, no salir de noche)?

9

¿Siente temor de transitar por algunas calles o zonas de la ciudad (colonias, barrios o pueblo)?
$868 \quad 614 \quad 254$

$(76,5) \quad(70,7) \quad(29,3)$

0,047

855

$(75,6)$

599

$(70,1)$

256

$(29,9)$

0,989

922

664

$(72,9)$

258

$(28,0)$
$(81,3)$

151

$(26,3)$

$5,902 *$

0,847

$(28,6)$ 
¿El contexto de violencia delincuencial que se vive en El Salvador

¿El ambiente de violencia delincuencial que se vive en El Salvador le produce nerviosismo e inseguridad?

12

le produce ansiedad y estrés (pánico, miedo, agitación, boca seca, inquietud, taquicardia)?

¿Considera usted que la situación de violencia delincuencial que vive el país le afecta el sueño?

¿El contexto de violencia delincuencial que se vive en El Salvador afecta su estabilidad emocional y mental?

\section{6}

$(76,4)$

961

$(84,8)$

751

$(65,9)$

807

$(70,7)$

\section{4}

$(70,9)$

$(29,1)$

674

$(70,1)$

(29.9)

$(29,9)$

216

$(28,8)$

$(71,2)$

571

$(70,8)$
236

$(29,2)$
0,001

0,965

$0,306 \dagger$

0,018

*p $<0,05$

En relación con los indicadores de problemas de salud mental y bienestar psicológico que frecuentemente presenta la muestra comparados por género, seguidamente se presentan las frecuencias y porcentajes más representativos del indicador de salud mental. En este sentido, se evalúa sí las preocupaciones le han hecho perder mucho sueño; $301(26,4 \%)$ expresaron que frecuentemente; de estos, $142(41,2 \%)$ son masculinos y $177(58,8 \%)$ son femeninos. Con relación a sí se ha sentido agobiado y con tensión, 345 $(30,5 \%)$ manifestaron que es frecuente; de estos, $143(41,4 \%)$ son masculinos y $202(58,6 \%)$ son del sexo femenino. Por otra parte, se preguntó si ha sentido que no puede superar sus dificultades; $347(30,6 \%)$ refieren que frecuentemente; de este total, $160(46,1 \%)$ son masculinos y $187(53,9 \%)$ son femeninos. En este mismo sentido, se evaluó si ha sido capaz de hacer frente a sus problemas, $917(80,7 \%)$ dicen que frecuentemente; hay $19,3 \%$ que no pueden con sus problemas. Del total, $439(47,9 \%)$ son masculinos y $478(52,1 \%)$ son femeninos. Con respecto a si se ha sentido poco feliz y deprimido, $244(21,5 \%)$ expresaron que frecuentemente; del total, $104(42,6 \%)$ son masculinos y 140 $(57,4 \%)$ son femeninos. En todos los indicadores de salud mental comparados por sexo se encontraron porcentajes mayores en las mujeres que en los hombres, lo que indica que existen más dificultades de salud mental/bienestar psicológico en las mujeres.
En cuanto a si las preocupaciones le han hecho perder mucho sueño, 301 (26,4\%) expresaron que frecuentemente; de estos, $202(67,1 \%)$ son urbanos y $99(32,9 \%)$ son rurales. Referente a sí se ha sentido constantemente agobiado y con tensión, 345 (30.5\%) manifestaron que sí; de este total, $252(73,0 \%)$ son residentes urbanos y $93(27,0 \%)$ son residentes rurales. Al preguntarles si han sentido que no pueden superar sus dificultades, $347(30,6 \%)$ refieren que frecuentemente; de estos, $230(66,3 \%)$ son urbanos y $117(33,7 \%)$ son rurales. Por otra parte, se preguntó sí se han sentido poco feliz y deprimido $244(21,5 \%)$ expresaron que frecuentemente, de este total, $161(66,0 \%)$ son residentes urbanos y $83(34,0 \%)$ son residentes rurales. En cuanto a si se sienten razonablemente felices, considerando todas las circunstancias, $756(67,1 \%)$ manifestaron que frecuentemente; de estos, 537 (71,0\%) son urbanos y 219 $(29,0 \%)$ son rurales. Este último indicador refiere entonces que el $32,9 \%$ de la muestra del estudio no se considera feliz, considerando todas las circunstancias. En un sentido general, se puede apreciar que en todos los indicadores de salud mental y bienestar psicológico de la población, en función del lugar de residencia, existe mayor incidencia de dificultades de salud mental en la muestra de residentes urbanos que en la muestra de residentes rurales, duplicando los porcentajes en casi todos los indicadores. 


\section{Discusión}

Para elaborar la discusión de los resultados en este informe se hará énfasis en aquellos hallazgos más destacados y que estén en sintonía con los objetivos del estudio. En un primer momento, se tiene que más de una tercera parte $(36,0 \%)$ de la muestra estudiada manifestó que un pariente suyo fue víctima de la violencia delincuencial (lesionado o asesinado) en el último año. Estos resultados están en sintonía con los encontrados por el Centro de Investigación de la Opinión Pública Salvadoreña (Ciops, 2011), que informó que la organización criminal y los tipos de delitos que se cometen en el lugar de residencia por la delincuencia (pandillas) es de $30,9 \%$; los tipos de delitos son $23,5 \%$ hurtos y robos, y el $18,8 \%$ dijo que era la extorsión. Así mismo, un $36,1 \%$ manifestó haber sido víctima de robo en la calle. Por otra parte, el estudio revela que una inmensa mayoría de la población salvadoreña $(76,5 \%)$ considera que las noticias que se transmiten por televisión, prensa escrita y la radio, sobre la violencia delincuencial (lesionados, muertos, robos, extorciones, etc.) afectan su estado emocional y mental. La población expresa tener miedo y temor $(81,3 \%)$ de transitar por algunas calles, colonias, barrios y pueblos, de ser víctima de la violencia social delincuencial. En esta misma dirección, más de tres cuartas partes $(76,4 \%)$ de la muestra evaluada manifestó que el contexto de violencia social delincuencial en que vive le produce ansiedad y estrés (pánico, miedo, agitación, boca seca, inquietud y taquicardia). Hay que destacar también que el ambiente de violencia social delincuencial que viven los salvadoreños les produce nerviosismo e inseguridad (84,8\%). De igual forma, esta situación les afecta el sueño (65,9\%). El contexto sociocultural de violencia delincuencial que viven los salvadoreños está afectando su estabilidad emocional y mental (70,7\%). En todas las variables se encontró una prevalencia de estrés $y$ ansiedad delincuencial mayor en las mujeres que en los hombres. Los resultados anteriores coinciden con la descripción del estrés que hace Seyle (1936), como un síndrome específico constituido por cambios inespecíficos del organismo, inducidos por las demandas del entorno. El estrés es una respuesta inespecífica del organismo ante una diversidad de exigencias. Se trata de un proceso adaptativo y de emergencia, siendo imprescindible para la supervivencia de la persona. Lazarus y Folkman (1986) describen que el estrés es una relación entre el individuo y su entorno, que es evaluado por aquel como amenazante, y que pone en peligro su bienestar. Un indicador importante de señalar en este análisis es que existe una prevalencia más alta de estrés y ansiedad delincuencial en la población que reside en las ciudades, en comparación con las personas que residen en el área rural. Sin embargo, hay que señalar que el contexto de violencia social delincuencial afecta significativamente a la población general, pero de forma más pronunciada a los que residen en las ciudades. En consecuencia, lo antes señalado conformaría un factor psicosocial que afecta la salud mental y bienestar psicológico en la población salvadoreña.

Estos hallazgos coinciden con los encontrados por Lederbogen, Kirsch, Haddad, Streit, Schuch, Tost... MeyerLindenberg (2011), quienes expresan que las personas que viven en la ciudad, a diferencia de otras personas, tienen más probabilidades de sufrir más estrés y enfermedades mentales. Estos resultados se basan en la investigación alemana que compara patrones de actividad cerebral en respuesta al estrés social de los habitantes urbanos y rurales. Los autores plantean que estudios anteriores han demostrado que los problemas de salud mental, tales como esquizofrenia, ansiedad y trastornos del humor, son generalmente más comunes en personas que viven o crecen en las ciudades. El equipo investigador informó que anteriores estudios epidemiológicos han demostrado que los residentes urbanos tienen un riesgo mucho mayor de trastornos psicológicos, incluyendo los trastornos de ansiedad, depresión y esquizofrenia. La salud mental. En este estudio se encontró que más de una cuarta parte $(26,4 \%)$ de la muestra en estudio refiere que las preocupaciones le han hecho perder mucho sueño; en esta misma dirección, $30,5 \%$ expresa que se ha sentido agobiado y con tensión, una prevalencia similar $(30,6 \%)$ siente que no puede superar sus dificultades; cerca de una quinta parte $(19,3 \%)$ dice que ha sido incapaz de hacer frente a sus problemas.

\section{Referencias}

Acevedo, C. (2008). "Los costos económicos de la violencia en El Salvador". América Latina Hoy, Vol. 50, 71-88.

Adler, N. y Matthews, K. (1994). "Health Psychology: why do some people get sick and some stay well?". Annual Review of Psychology, 45, pp. 229-259. 
Alejo, E. G. (2005). Aplicación del modelo de estrés postraumático en el estudio del impacto de la violencia sobre la salud mental en población desplazada. Universidad Autónoma de Bucaramanga, Colombia. Disponible en http://www.editorial. unab.edu.co/revistas/medunab

American Psychiatric Association (APA). (1986). Diagnostic and statistical manual of mental disorders (DSM-II) (2nd Ed.) Washington, DC: Author.

American Psychiatric Association (APA). (1994). Diagnostic and stastical manual of mental disorders (DSM-IV) (4th. Ed.). Washington, DC: Author.

American Psychological Association (APA). (2010). Manual de publicaciones, (3ra. Ed.). Editorial. Manual modern, México.

Ansorena, A.; Cobo, J. \& Romero, I. (1983). El constructo de ansiedad en psicología". Estudios de Psicología, 16, pp. 31-45.

Axelsson, L. \& Ejlertsson, G. (2002). Self-reported health, selfesteem and social support among young unemployed people: a population based study. Internation Journal of Social Welfare, 11, pp. 11-119.

Barcelata, B. \& Álvarez, I. (2005). Patrones de interacción familiar de madres y padres generadores de violencia y maltrato infantil. Acta colombiana de psicología, 8, 35-46.

Begotti, T., Borca, G.; Calandri, E.; Cattelino, E; Ingoglia, S. (2004). Family relationships and risk: an análisis of circularity. IX Conference of the European Association for Research on Adolescence. 0 Porto.
Barraca, J. \& López L. (1997). Escala de satisfacción familiar. Madrid: TEA.

Bagley, C. \& Mallick, K. (2001). Normative data and mental health construct validity for the Rosenberg Self-Esteem Scale in British Adolescents. International Journal of Adolescence and Youth, 9, pp. 117-126.

Banco Mundial (BM). (2011). Crimen y violencia en Centroamérica. Un desafío para el desarrollo. Departamento de desarrollo sostenible y reducción de la pobreza y gestión económica, región de América Latina y el Caribe.

Barnes, H. L., (Olson, D.H. 1982). Parent-adolescent communication scale. En: Olson DH, McCubbin $\mathrm{H}$, Barnes $\mathrm{H}$, Larsen A, Muxen $\mathrm{M}$, Wilson W (eds). Family Inventories: Inventories Used in a National Survey of Families across the Family Life Cycle. University of Minnesota Press, 33-48, St. Paul.

Bermúdez, J. \& Luna, M. D. (1980). Ansiedad. In Fernández, F.J., Trespalacios (Ed.), Psicología general II (pp. 195-213). Madrid: UNED.

Borkevek, T. D.; Weerts, T. C. \& Berstein, D. A. (1977). Assesment of anxiety. In Ciminero, A. R., Calhon, K. S., y Adams, H. E. (Eds.). Handbook of Behavioral Assesment (pp. 353-403). New York: John Wiley.

Bronfenbrenner, U. (1979). The ecology of Human Development. Cambridge, Harvard University Press. (Trad. Cast.): La ecología del desarrollo humano. Barcelona, Ediciones Paidós, 1987). 